\title{
Minireview \\ MicroRNAs in cell proliferation, cell death, and tumorigenesis
}

\author{
H-W Hwang' and JT Mendell ${ }^{*, I}$ \\ 'Program in Human Genetics and Molecular Biology, The Institute of Genetic Medicine, Johns Hopkins University School of Medicine, 733 N Broadway, \\ BRB 460C, Baltimore, MD 21205, USA
}

\begin{abstract}
MicroRNAs (miRNAs) are a recently discovered class of $\sim 18-24$ nucleotide RNA molecules that negatively regulate target mRNAs. All studied multicellular eukaryotes utilise miRNAs to regulate basic cellular functions including proliferation, differentiation, and death. It is now apparent that abnormal miRNA expression is a common feature of human malignancies. In this review, we will discuss how miRNAs influence tumorigenesis by acting as oncogenes and tumour suppressors.

British Journal of Cancer (2006) 94, 776-780. doi:I0.I038/sj.bjc.6603023 www.bjcancer.com

Published online 2I February 2006

(c) 2006 Cancer Research UK
\end{abstract}

Keywords: microRNAs; cellular proliferation; apoptosis; oncogene; tumour suppressor

MicroRNAs (miRNAs) were first discovered in the early 1990s by Ambros, Ruvkun, and co-workers while studying development in the nematode Caenorhabditis elegans (Lee et al, 1993; Wightman et al, 1993). Their analyses of genes that control developmental timing revealed that lin-14 was necessary for the differentiation of specific cell lineages and lin- 4 might be a trans-acting negative regulator of $l i n-14$. Subsequently, it was determined that lin-4 encodes a 22-nucleotide noncoding RNA which negatively regulates the translation of lin-14 by directly base pairing to complementary sites within its $3^{\prime}$-untranslated region ( $3^{\prime}$ UTR). Since very few examples of regulation of an mRNA by a naturally occurring antisense RNA had been described in eukaryotes, this form of regulation was generally considered a fascinating, but likely worm-specific, oddity. Seven years later, another small regulatory RNA, let-7, was identified as an additional regulator of developmental timing in C. elegans (Reinhart et al, 2000). Strikingly, both the sequence and temporal expression pattern of let-7 were conserved in a wide variety of animals (Pasquinelli et al, 2000), suggesting a more general role for small RNAs in gene regulation. Nevertheless, the broad significance of these findings was not fully appreciated until hundreds of similar small RNAs were identified in C. elegans, Drosophila, and mammals by molecular cloning and bioinformatics analyses in 2001 (reviewed in Bartel, 2004). These newly discovered $\sim 18-24$ nucleotide RNAs were termed microRNAs (miRNAs). Since their initial description, more than 3000 miRNAs have been identified in animals, plants, and viruses. In the human genome, more than 300 miRNAs have been discovered, and the estimated number of miRNA genes is as high as 1000 (Bentwich et al, 2005; Berezikov et al, 2005). As a group, miRNAs are estimated to regulate $30 \%$ of genes in the human genome (Lewis et al, 2005).

In addition to providing critical functions during normal development and cellular homeostasis, it has become increasingly clear that abnormalities in miRNA activity contribute to human disease pathogenesis. In particular, an important role for miRNAs

*Correspondence: Dr JT Mendell; E-mail: jmendell@jhmi.edu Received 22 November 2005; revised 30 January 2006; accepted 31 January 2006; published online 2I February 2006 in the development of cancer has emerged. In this review, we will discuss the functions of cancer-relevant miRNAs that regulate cellular proliferation, death, and tumorigenesis.

\section{miRNA BIOGENESIS AND FUNCTION}

MicroRNAs are initially transcribed by RNA polymerase II (pol II) as long primary transcripts known as primary-miRNAs (primiRNAs). Like other pol II transcripts, these RNAs are capped, polyadenylated, and are usually several kilobases in length (reviewed in Kim, 2005). Approximately half of pri-miRNAs appear to lack significant open reading frames and are likely noncoding RNAs. Nevertheless, these transcripts are often spliced and many miRNAs are located within their intronic segments. Alternatively, a significant fraction of miRNAs $(\sim 40 \%)$ are found within introns of known protein-coding genes (Rodriguez et al, 2004). Additionally, miRNAs are frequently clustered such that a single pri-miRNA contains multiple miRNA sequences.

Within the pri-miRNA, the miRNA itself is contained within a $\sim 60-80$ nucleotide sequence that can fold back on itself to form a stem-loop hairpin structure. MicroRNA hairpins are recognised and excised from pri-miRNAs in the nucleus (Figure 1) by the microprocessor complex, which includes the RNase III enzyme drosha and its binding partner DGCR8 (Kim, 2005). The excised miRNA hairpins, referred to as pre-miRNAs, contain a two nucleotide $3^{\prime}$ overhang characteristic of RNase III cleavage products. Pre-miRNAs are rapidly exported to the cytoplasm by the nuclear export factor exportin 5 which uses Ran-GTP as a cofactor (Yi et al, 2003; Bohnsack et al, 2004; Lund et al, 2004). Further cytoplasmic processing by dicer, a second RNase III enzyme, produces an $\sim 18-24$ nucleotide duplex. This fully processed duplex is incorporated in an ATP-independent manner into a large protein complex known as the RNA-induced silencing complex, or RISC, which includes as core components the Argonaute proteins (Agol-4 in humans). Only one strand of the miRNA duplex remains stably associated with RISC. This strand becomes the mature miRNA. The opposite strand, known as the passenger strand or miRNA*, is disposed of through two alternative mechanisms. When miRNAs are loaded into RISC 


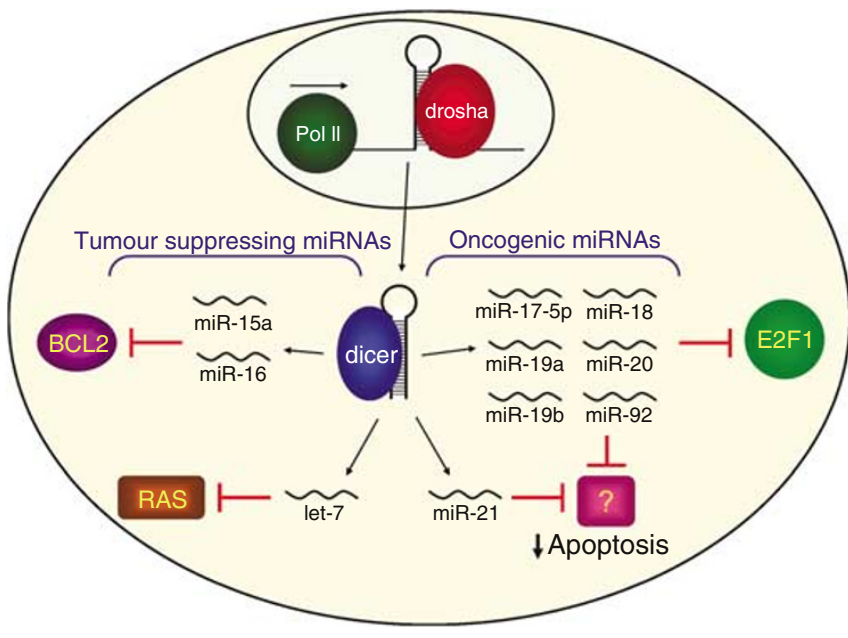

Figure I The biogenesis and functions of cancer-associated human miRNAs. A simplified schematic of miRNA biogenesis is shown. MicroRNAs are initially transcribed by RNA polymerase $\|$ (Pol II) and sequentially processed by drosha and dicer (see text for details). MicroRNAs with tumour suppressing or oncogenic properties are shown with their experimentally validated targets.

containing Ago2, the only human Ago protein capable of cleaving target mRNAs, the passenger strand may be cleaved. Alternatively, RISC containing any Ago protein may remove the passenger strand via a bypass mechanism that does not require cleavage and likely involves duplex unwinding (Gregory et al, 2005; Matranga et al, 2005; Rand et al, 2005).

MicroRNAs act as guides that direct RISC to target mRNAs that are subsequently cleaved or translationally silenced (reviewed in Bartel, 2004). The degree of complementarity between an miRNA and its target determines the mechanism of regulation. When an miRNA and an mRNA exhibit perfect complementarity, the target mRNA is cleaved by RISC. This appears to be the predominant mechanism through which miRNAs function in plants. Imperfect base pairing between an miRNA and its target, as occurs with most C. elegans, Drosophila, and mammalian miRNAs, leads to translational silencing of the target. However, imperfectly complementary miRNAs can also reduce the abundance of target mRNAs to varying degrees (He and Hannon, 2004).

\section{EARLY CLUES THAT miRNAs REGULATE CANCER-RELEVANT PATHWAYS}

Malignant cell growth is typified by loss of cellular identity, enhanced proliferation, and dysregulated cell death. Early clues that miRNAs were important in regulating these cancer-relevant cellular phenotypes came from genetic screens in C. elegans and Drosophila. As mentioned above, the first miRNA identified, lin-4, controls the timing of developmental events in C. elegans. Loss-offunction of lin-4 results in the abnormal differentiation of specific cell lineages and the reiteration of embryonic cell types in later stages of development (Lee et al, 1993). These abnormalities are reminiscent of tumours which are caused by a failure to fully execute a programme of differentiation. For example, in many haematologic malignancies, a block in differentiation causes immature cells to accumulate with pathologic consequences.

In Drosophila, overexpression of the Bantam miRNA causes overgrowth of wing and eye tissue. It was subsequently demonstrated that Bantam stimulates cell proliferation and prevents apoptosis at least in part through negative regulation of the proapoptotic gene hid (Brennecke et al, 2003). Another Drosophila miRNA, miR-14, was identified through its ability to suppress cell death. Flies harbouring a deletion of this miRNA were found to have an increased expression of an apoptotic effector caspase, Drice, which has a putative miR-14 target site (Xu et al, 2003). However, direct regulation of Drice by miR-14 remains to be demonstrated. In another study, antisense-mediated inhibition of the miR-2/6/11/13/308 family in Drosophila embryos was demonstrated to induce widespread apoptosis. These miRNAs were subsequently shown to regulate the proapoptotic factors hid, grim, reaper, and sickle (Leaman et al, 2005).

\section{miRNA DYSREGULATION IN CANCER}

A more direct link between miRNA function and cancer pathogenesis is supported by studies examining the expression of miRNAs in clinical samples. The first study documenting abnormalities in miRNA expression in tumour samples focused on B-cell chronic lymphocytic leukaemia (B-CLL). Deletion of chromosome 13q14 is the most frequent chromosomal abnormality in this disorder and it has been postulated that a tumour suppressor gene resides in this region. Croce and co-workers demonstrated that this tumour suppressor activity is likely provided by two miRNAs, miR-15a and miR-16-1, which are localised in the minimally deleted region and are frequently deleted or downregulated in CLL patients (Calin et al, 2002). More recently, these investigators found that a specific expression signature consisting of 13 miRNAs, including miR-15a and miR16-1, was associated with disease progression in CLL (Calin et al, 2005).

More than half of miRNAs are located at sites in the human genome that are frequently amplified, deleted, or rearranged in cancer, suggesting that miRNA abnormalities play a broad role in cancer pathogenesis (Calin et al, 2004). Also consistent with this notion is the observed dysregulation of miRNA expression in diverse cancer subtypes including Burkitt's lymphoma (Metzler et al, 2004), colorectal cancer (Michael et al, 2003), lung cancer (Takamizawa et al, 2004), breast cancer (Iorio et al, 2005), and glioblastoma (Chan et al, 2005).

Recently, Golub and co-workers used a bead-based flow cytometric method to profile 217 mammalian miRNAs across a large panel of samples representing diverse human tissues and tumours (Lu et al, 2005). They found that miRNA profiles were highly informative, reflecting the developmental lineage and differentiation state of the tumours. MicroRNA profiles were used to identify the tissue origin of poorly differentiated tumours with greater accuracy than profiles constructed using $\sim 16000$ mRNAs. Furthermore, tumours within the same lineage harbouring distinct chromosomal rearrangements were found to exhibit distinct miRNA expression profiles. Thus, the developmental history of a tumour is reflected in its miRNA expression pattern.

Irrespective of cell type, more than half of the miRNAs examined in the Golub study were expressed at significantly lower levels in tumours compared with normal tissues. This likely reflects a role for miRNAs in terminal differentiation and the relatively incomplete differentiation status of cancer cells. Consistent with this hypothesis, inducing differentiation of a myeloid leukaemia cell line with all-trans retinoic acid increased the expression of many miRNAs. A similar induction of miRNA expression was also observed in primary human haematopoietic progenitor cells undergoing erythroid differentiation.

The emerging view from these studies is that dysregulation of miRNA expression is a frequent occurrence in diverse types of cancer. These findings highlight the potential utility of miRNA profiling for diagnostic and prognostic applications. These studies also highlight a need for more direct functional analyses of the roles of miRNAs in regulating pathways relevant to tumour pathogenesis. These types of experiments are now beginning to be carried out, as described below. 


\section{PROPROLIFERATIVE/ANTIAPOPTOTIC miRNAs}

MicroRNAs with proproliferative and antiapoptotic activity would likely promote oncogenesis and thus may be overexpressed in cancer cells. The most comprehensively studied example of an miRNA locus with these properties is the mir-17 cluster, consisting of six miRNAs: miRs-17-5p, -18, -19a, -19b, -20, and -92. This miRNA cluster is located on human chromosome 13q31, a region that is frequently amplified in several types of lymphoma and solid tumours (Ota et al, 2004). In a recent study, He et al (2005) found that miRNAs from this cluster are highly expressed in B-cell lymphoma samples and cell lines. To directly interrogate the tumorigenic properties of these miRNAs, they utilised $\mathrm{E} \mu$-Myc transgenic mice, a model of B-cell lymphoma in which expression of the proto-oncogene c-Myc is driven by the immunoglobulin heavy-chain enhancer. c-Myc encodes a helix-loop-helix transcription factor that is abnormally active in a large fraction of human malignancies. Elevated c-Myc expression accelerates cell proliferation and growth and, in some settings, induces apoptosis. $\mathrm{E} \mu$-Myc transgenic mice develop B-cell lymphomas relatively late in life (4-6 months of age) and with incomplete penetrance. When a truncated form of the mir-17 cluster (lacking miR-92) was introduced into haematopoietic stem cells from these mice, disease onset was dramatically accelerated. Whereas $\mathrm{E} \mu$-Myc lymphomas are highly apopotic, the miRNA-expressing lymphomas show a high mitotic index without extensive apoptosis, suggesting that these miRNAs act primarily by suppressing cell death. Interestingly, when each of the miRNAs of the mir-17 cluster was individually coexpressed with $\mathrm{c}-\mathrm{Myc}$, none accelerated tumour formation, suggesting that the oncogenic effect requires a cooperative interaction between the miRNAs in the cluster. Moreover, no increased lymphomagenesis was observed after expressing 96 different single miRNAs in this model, demonstrating the specificity of the mir-17 cluster finding.

While a functional interaction between the mir-17 cluster and Myc was being explored in the $\mathrm{E} \mu$-Myc animals, independent work by O'Donnell et al (2005) discovered that these miRNAs were directly regulated at the transcriptional level by c-Myc. In multiple cellular models, induction of c-Myc expression correlated with induction of the mir-17 cluster. Furthermore, c-Myc was found to bind directly to the genomic locus encoding these miRNAs to activate their transcription. The authors went on to show that two miRNAs in this cluster, miR-17-5p and miR-20, regulate the translational efficiency of the proproliferative/proapoptotic transcription factor E2F1. Interestingly, E2F1 is known to be transcriptionally activated by c-Myc (Fernandez et al, 2003). Since high expression of E2F1 is sufficient to induce apoptosis in some settings, simultaneous transcriptional activation by c-Myc and dampening of translational efficiency by the mir-17 cluster may tune E2F1 activity such that cell death is disfavoured and proliferation is promoted. This hypothesis is consistent with the decreased apoptosis observed in the $\mathrm{E} \mu$-Myc miRNA-expressing tumours.

Amplification of the mir-17 cluster and overexpression of these miRNAs has more recently been observed in lung cancers and in particular small-cell lung cancer (Hayashita et al, 2005). In a lung cancer cell line, overexpression of the entire mir-17 cluster, but not individual miRNAs from the cluster, accelerated cell proliferation. These findings further document the oncogenic properties of this group of miRNAs.

Another miRNA with an apparent antiapoptotic function is miR-21. This miRNA was found to be overexpressed in glioblastoma tumour tissues and cell lines (Chan et al, 2005). High expression of this miRNA was also observed in breast cancer tissue (Iorio et al, 2005). Inhibition of miR-21 in glioblastoma cell lines using 2'-O-methyl antisense oligonucleotides, which are known to inhibit miRNA function, resulted in caspase activation and increased apoptosis (Chan et al, 2005).

\section{ANTIPROLIFERATIVE/PROAPOPTOTIC miRNAs}

MicroRNAs with antiproliferative and proapoptotic activity are likely to function as tumour suppressor genes and thus may be underexpressed in cancer cells. The family of let-7 miRNAs represents a clear example of this. The let-7 family consists of a group of highly conserved miRNAs in multiple species including $C$. elegans, Drosophila, and vertebrates. let-60/RAS was recently identified as a target of let-7 in C. elegans (Johnson et al, 2005). This regulation appears to be conserved in humans where three RAS genes, known to be potent oncogenes, have also been demonstrated to be directly regulated by human let-7. Since RAS dysregulation is a key oncogenic event in lung cancer, loss of let-7 may contribute to pathogenesis in this disorder. Indeed, let-7 is generally expressed at low levels in cancerous lung tissue compared to normal tissue and low expression of this miRNA correlates with shorter postoperative lung cancer survival (Takamizawa et al, 2004). Furthermore, in samples from three lung cancer patients, RAS protein levels, but not RAS mRNA levels, were inversely correlated with let-7 levels, consistent with miRNAmediated translational repression (Johnson et al, 2005). Expression of let-7 in lung cancer cell lines directly suppresses growth in vitro, further documenting the tumour suppressing activity of this miRNA (Takamizawa et al, 2004).

miR-15a and miR-16-1 represent additional miRNAs with likely tumour suppressing activity. As mentioned above, these miRNAs are located on human chromosome 13 in a region frequently deleted in B-CLL. Cimmino et al (2005) identified a conserved target site for miR-15a and miR-16-1 in the $3^{\prime}$ UTR of BCL2, a potent inhibitor of cell death. Consistent with a regulatory interaction between these miRNAs and this gene, the levels of miR-15a and miR-16-1 are inversely correlated with Bcl2 protein levels in samples from CLL patients. Furthermore, overexpression of miR-15a and miR-16-1 in a leukaemic cell line results in decreased Bcl2 protein expression, activation of the intrinsic apoptosis pathway, and ultimately cell death. Thus, loss of these miRNAs may contribute to elevated $\mathrm{Bcl} 2$ expression and pathologic cell survival in B-CLL.

\section{miRNAs IN CELL CYCLE PROGRESSION AND STEM CELL MAINTENANCE}

Both cancer cells and stem cells share an ability to divide continuously without undergoing senescence. Additionally, evidence supports the existence of 'cancer stem cells' that may be important in the genesis of certain types of cancers including leukaemia and solid tumours. Because of the importance of these cells in normal and pathophysiologic states, there is growing interest in elucidating the mechanisms that confer the unique properties of these cells. Recent experiments in which components essential for miRNA biogenesis were disrupted in flies suggest that miRNAs may play a role in stem cell maintenance. Drosophila with a null mutation of dicer-1 (dcr-1), which is required for miRNA processing, exhibited abnormalities in germline stem cell (GSC) function (Hatfield et al, 2005). Specifically, dcr-1-deficient GSCs inappropriately activated the G1/S cell cycle checkpoint, suggesting that miRNAs normally act to promote continuous cell cycle progression. This cell cycle delay was specific to GSCs and did not occur in other tissues such as imaginal discs. Reduction of expression of Dacapo (Dap), a Drosophila homologue of the p21/ p27 family of cyclin-dependent kinase inhibitors, partially rescued the dcr-1 deficiency phenotype. Dap is predicted to be regulated by multiple miRNAs and expression of a Dap transgene lacking these putative miRNA-binding sites mimicked the phenotype of dcr-1 loss-of-function. Taken together, these data suggest that miRNAs normally function to suppress Dap expression in GSCs, thus allowing cell-cycle progression. 


\section{CONCLUDING REMARKS}

It is now well established that miRNAs play essential roles in many basic physiologic processes including proliferation, differentiation, and apoptosis. Abnormalities in miRNA function impinge upon the normal regulation of these pathways and thus influence tumorigenesis. Undoubtedly, the growing interest in this class of regulatory RNAs will lead to continued classification of miRNA expression in cancer samples and the identification of new miRNAs that may act as oncogenes and tumour suppressors. The key to understanding the roles of these molecules in cancer will be identifying and validating the critical targets responsible for the phenotypic effects of miRNA loss- or gain-of-function. Although bioinformatics approaches have produced exhaustive lists of putative targets, verifying these regulatory interactions and determining which are the critical targets in a given tissue or tumour remains arduous.

Another potential challenge for future studies relates to the probable tissue-specific functions of some miRNAs. The function of a given miRNA is dictated by the milieu of targets that are expressed in a given cell type. Thus, an miRNA that regulates both proproliferative and antiproliferative targets may act as a tumour suppressor in some cancers and an oncogene in others, depending on which targets are driving tumorigenesis. One example of this may be the mir-17 cluster. The evidence that these miRNAs have

\section{REFERENCES}

Bartel DP (2004) MicroRNAs: genomics, biogenesis, mechanism, and function. Cell 116: $281-297$

Bentwich I, Avniel A, Karov Y, Aharonov R, Gilad S, Barad O, Barzilai A, Einat P, Einav U, Meiri E, Sharon E, Spector Y, Bentwich Z (2005) Identification of hundreds of conserved and nonconserved human microRNAs. Nat Genet 37: 766-770

Berezikov E, Guryev V, van de Belt J, Wienholds E, Plasterk RH, Cuppen E (2005) Phylogenetic shadowing and computational identification of human microRNA genes. Cell 120: 21-24

Bohnsack MT, Czaplinski K, Gorlich D (2004) Exportin 5 is a RanGTPdependent dsRNA-binding protein that mediates nuclear export of premiRNAs. RNA 10: 185-191

Brennecke J, Hipfner DR, Stark A, Russell RB, Cohen SM (2003) Bantam encodes a developmentally regulated microRNA that controls cell proliferation and regulates the proapoptotic gene hid in Drosophila. Cell 113: $25-36$

Calin GA, Dumitru CD, Shimizu M, Bichi R, Zupo S, Noch E, Aldler H, Rattan S, Keating M, Rai K, Rassenti L, Kipps T, Negrini M, Bullrich F, Croce CM (2002) Frequent deletions and down-regulation of micro-RNA genes miR15 and miR16 at 13q14 in chronic lymphocytic leukemia. Proc Natl Acad Sci USA 99: 15524-15529

Calin GA, Ferracin M, Cimmino A, Di Leva G, Shimizu M, Wojcik SE, Iorio MV, Visone R, Sever NI, Fabbri M, Iuliano R, Palumbo T, Pichiorri F, Roldo C, Garzon R, Sevignani C, Rassenti L, Alder H, Volinia S, Liu CG, Kipps TJ, Negrini M, Croce CM (2005) A microRNA signature associated with prognosis and progression in chronic lymphocytic leukemia. $N$ Engl $J$ Med 353: $1793-1801$

Calin GA, Sevignani C, Dumitru CD, Hyslop T, Noch E, Yendamuri S, Shimizu M, Rattan S, Bullrich F, Negrini M, Croce CM (2004) Human microRNA genes are frequently located at fragile sites and genomic regions involved in cancers. Proc Natl Acad Sci USA 101: 2999-3004

Chan JA, Krichevsky AM, Kosik KS (2005) MicroRNA-21 is an antiapoptotic factor in human glioblastoma cells. Cancer Res 65: 60296033

Cimmino A, Calin GA, Fabbri M, Iorio MV, Ferracin M, Shimizu M, Wojcik SE, Aqeilan RI, Zupo S, Dono M, Rassenti L, Alder H, Volinia S, Liu CG, Kipps TJ, Negrini M, Croce CM (2005) miR-15 and miR-16 induce apoptosis by targeting BCL2. Proc Natl Acad Sci USA 102: $13944-13949$

Fernandez PC, Frank SR, Wang L, Schroeder M, Liu S, Greene J, Cocito A, Amati B (2003) Genomic targets of the human c-Myc protein. Genes Dev 17: $1115-1129$ oncogenic activity was presented earlier in this review. Nevertheless, the chromosomal locus encompassing these miRNAs is known to undergo loss-of-heterozygosity in hepatocellular carcinoma (Lin et al, 1999), suggesting that these miRNAs may have tumour suppressor activity in this type of cancer. Additionally, many proproliferative targets are predicted to be downregulated by these miRNAs. Future studies are necessary to determine if these and other miRNAs have cell-type-specific effects on cell proliferation and death.

Although we are at an early stage in our understanding of the roles of miRNAs in cancer, the importance of these molecules in this disease process are clear. Undoubtedly, continued efforts to delineate miRNA function in physiologic and pathophysiologic states will reveal novel insights into normal cellular and developmental biology and the mechanisms that are disrupted when these processes go awry.

\section{ACKNOWLEDGEMENTS}

We thank Kate O'Donnell and members of the Mendell laboratory for critical reading of the manuscript. We apologise to those whose work was not cited due to space limitations. JTM is a March of Dimes Basil O’Connor Scholar.
Gregory RI, Chendrimada TP, Cooch N, Shiekhattar R (2005) Human RISC couples microRNA biogenesis and posttranscriptional gene silencing. Cell 123: $631-640$

Hatfield SD, Shcherbata HR, Fischer KA, Nakahara K, Carthew RW, Ruohola-Baker H (2005) Stem cell division is regulated by the microRNA pathway. Nature 435: 974-978

Hayashita Y, Osada H, Tatematsu Y, Yamada H, Yanagisawa K, Tomida S, Yatabe Y, Kawahara K, Sekido Y, Takahashi T (2005) A polycistronic microRNA cluster, miR-17-92, is overexpressed in human lung cancers and enhances cell proliferation. Cancer Res 65: 9628-9632

He L, Hannon GJ (2004) MicroRNAs: small RNAs with a big role in gene regulation. Nat Rev Genet 5: 522-531

He L, Thomson JM, Hemann MT, Hernando-Monge E, Mu D, Goodson S, Powers S, Cordon-Cardo C, Lowe SW, Hannon GJ, Hammond SM (2005) A microRNA polycistron as a potential human oncogene. Nature 435: $828-833$

Iorio MV, Ferracin M, Liu CG, Veronese A, Spizzo R, Sabbioni S, Magri E, Pedriali M, Fabbri M, Campiglio M, Menard S, Palazzo JP, Rosenberg A, Musiani P, Volinia S, Nenci I, Calin GA, Querzoli P, Negrini M, Croce CM (2005) MicroRNA gene expression deregulation in human breast cancer. Cancer Res 65: 7065-7070

Johnson SM, Grosshans H, Shingara J, Byrom M, Jarvis R, Cheng A, Labourier E, Reinert KL, Brown D, Slack FJ (2005) RAS is regulated by the let-7 microRNA family. Cell 120: 635-647

Kim VN (2005) MicroRNA biogenesis: coordinated cropping and dicing. Nat Rev Mol Cell Biol 6: 376-385

Leaman D, Chen PY, Fak J, Yalcin A, Pearce M, Unnerstall U, Marks DS, Sander C, Tuschl T, Gaul U (2005) Antisense-mediated depletion reveals essential and specific functions of microRNAs in Drosophila development. Cell 121: $1097-1108$

Lee RC, Feinbaum RL, Ambros V (1993) The C. elegans heterochronic gene lin-4 encodes small RNAs with antisense complementarity to lin-14. Cell 75: $843-854$

Lewis BP, Burge CB, Bartel DP (2005) Conserved seed pairing, often flanked by adenosines, indicates that thousands of human genes are microRNA targets. Cell 120: 15-20

Lin YW, Sheu JC, Liu LY, Chen CH, Lee HS, Huang GT, Wang JT, Lee PH, $\mathrm{Lu}$ FJ (1999) Loss of heterozygosity at chromosome 13q in hepatocellular carcinoma: identification of three independent regions. Eur J Cancer 35: $1730-1734$

Lu J, Getz G, Miska EA, Alvarez-Saavedra E, Lamb J, Peck D, Sweet-Cordero A, Ebert BL, Mak RH, Ferrando AA, Downing JR, Jacks T, Horvitz HR, 
Golub TR (2005) MicroRNA expression profiles classify human cancers. Nature 435: $834-838$

Lund E, Guttinger S, Calado A, Dahlberg JE, Kutay U (2004) Nuclear export of microRNA precursors. Science 303: 95-98

Matranga C, Tomari Y, Shin C, Bartel DP, Zamore PD (2005) Passengerstrand cleavage facilitates assembly of siRNA into Ago2-containing RNAi enzyme complexes. Cell 123: 607-620

Metzler M, Wilda M, Busch K, Viehmann S, Borkhardt A (2004) High expression of precursor microRNA-155/BIC RNA in children with Burkitt lymphoma. Genes Chromosomes Cancer 39: 167-169

Michael MZ, O'Connor SM, van Holst Pellekaan NG, Young GP, James RJ (2003) Reduced accumulation of specific microRNAs in colorectal neoplasia. Mol Cancer Res 1: 882-891

O'Donnell KA, Wentzel EA, Zeller KI, Dang CV, Mendell JT (2005) c-Mycregulated microRNAs modulate E2F1 expression. Nature 435: 839-843

Ota A, Tagawa H, Karnan S, Tsuzuki S, Karpas A, Kira S, Yoshida Y, Seto M (2004) Identification and characterization of a novel gene, C13orf25, as a target for 13q31-q32 amplification in malignant lymphoma. Cancer Res 64: $3087-3095$

Pasquinelli AE, Reinhart BJ, Slack F, Martindale MQ, Kuroda MI, Maller B, Hayward DC, Ball EE, Degnan B, Muller P, Spring J, Srinivasan A, Fishman M, Finnerty J, Corbo J, Levine M, Leahy P, Davidson E, Ruvkun $\mathrm{G}$ (2000) Conservation of the sequence and temporal expression of let-7 heterochronic regulatory RNA. Nature 408: 86-89
Rand TA, Petersen S, Du F, Wang X (2005) Argonaute2 cleaves the anti-guide strand of siRNA during RISC activation. Cell 123: $621-629$

Reinhart BJ, Slack FJ, Basson M, Pasquinelli AE, Bettinger JC, Rougvie AE, Horvitz HR, Ruvkun G (2000) The 21-nucleotide let-7 RNA regulates developmental timing in Caenorhabditis elegans. Nature 403: 901-906

Rodriguez A, Griffiths-Jones S, Ashurst JL, Bradley A (2004) Identification of mammalian microRNA host genes and transcription units. Genome Res 14: $1902-1910$

Takamizawa J, Konishi H, Yanagisawa K, Tomida S, Osada H, Endoh $\mathrm{H}$, Harano T, Yatabe Y, Nagino M, Nimura Y, Mitsudomi T, Takahashi T (2004) Reduced expression of the let-7 microRNAs in human lung cancers in association with shortened postoperative survival. Cancer Res 64: 3753 - 3756

Wightman B, Ha I, Ruvkun G (1993) Posttranscriptional regulation of the heterochronic gene lin-14 by lin-4 mediates temporal pattern formation in C. elegans. Cell 75: 855-862

Xu P, Vernooy SY, Guo M, Hay BA (2003) The Drosophila microRNA miR14 suppresses cell death and is required for normal fat metabolism. Curr Biol 13: $790-795$

Yi R, Qin Y, Macara IG, Cullen BR (2003) Exportin-5 mediates the nuclear export of pre-microRNAs and short hairpin RNAs. Genes Dev 17: 3011 3016 